\title{
Prevention Beats Removal: Avoiding Stripe Artifacts from Current Variation in Particle Beam Microscopy Through Time-Resolved Sensing
}

\author{
Luisa Watkins ${ }^{1}$, Sheila Seidel ${ }^{1}$, Minxu Peng ${ }^{1}$, Akshay Agarwal ${ }^{2}$, Christopher $\mathrm{Yu}^{3}$ and Vivek Goyal ${ }^{4}$ \\ ${ }^{1}$ Boston University, United States, ${ }^{2}$ Massachusetts Institute of Technology, United States, ${ }^{3}$ Draper \\ Laboratory, United States, ${ }^{4}$ Boston University, Boston, Massachusetts, United States
}

Focused ion and electron beam microscopes suffer performance degradation when beam current deviates from the desired setting. When a sample is raster scanned across rows, fluctuations in the beam current may cause horizontal stripe artifacts to appear in the micrograph. The structure of these stripes depends heavily on the speed of beam current variation. Slow variation gives rise to thicker stripes that may span multiple rows, while faster variation results in short stripes that span a small fraction of a single row. Although source instability may be addressed through baking [1], this process can cost days of use on a valuable instrument. Existing computational mitigation techniques rely on signal processing tools to remove horizontal stripes from micrographs [2]. These methods need to be tuned to match the given scenario and work best when the beam current varies at a speed that gives rise to clear, wide horizontal stripes. Furthermore, they run the risk of reducing the contrast of horizontal features that actually appear in the sample. In this work, we demonstrate that the time-resolved (TR) processing methods described in [3] prevent artifacts due to beam current variation, regardless of their spatial structure.

Here we assume direct electron detection and adopt a quantitative approach to image formation that enables the use of probabilistic estimation methods. Even if the goals of the imaging are not quantitative, the qualitative improvement is apparent. In particle beam microscopy, the number of incident ions $M$ over dwell time $t$ is well-modeled as a Poisson random variable [4], with mean $\lambda=\Lambda t$, where $\Lambda$ is the rate of incident ions per unit time. The number of secondary electrons (SEs) emitted in response to each incident ion may be modeled as an independent Poisson random variable with parameter $\eta$, which is the mean SE yield per incident ion at that pixel and the quantity we seek to describe in our micrograph. We denote by $Y$ the total number of SEs observed over dwell time $t$. When $\lambda$ is known, the conventional estimator is given by: $\eta_{\text {baseline }}=\mathrm{Y} / \lambda$. Note that when $\lambda$ is imperfectly known, error propagates into

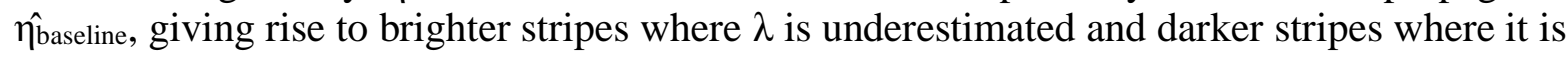
overestimated.

In [3], the concept of TR measurement is introduced. Dwell time $t$ is split into $n$ sub-acquisitions, each with mean dose $\lambda / n$, and $n$ SE measurements are collected: $Y_{1}, Y_{2}, . . Y_{n}$. Three different proposed estimators combine these $n$ measurements to form images of higher fidelity than $\eta_{\text {baseline, despite no }}$ increase of the total dose. When $\lambda / n$ is small, the probability of more than one incident ion during a single sub-acquisition becomes small. If $\eta$ is large enough that most incident ions result in at least $1 \mathrm{SE}$, the number of sub-acquisitions where the number of observed SEs is strictly positive may approximate the number of incident ions. The quotient mode estimator is inspired by this insight:

$$
\widehat{\eta}_{\mathrm{QM}}=\frac{Y_{1}+Y_{2}+\cdots+Y_{n}}{\sum_{k=1}^{n} \mathbb{1}_{\left\{Y_{k}>0\right\}}} .
$$


When $\eta$ is low, incident ions will more often expel no SEs, leading to an underestimate of $M$. The Lambert quotient mode (LQM) estimator seeks to correct for this phenomenon:

$$
\widehat{\eta}_{\mathrm{LQM}}=W\left(-\widehat{\eta}_{\mathrm{QM}} e^{-\widehat{\eta}_{\mathrm{QM}}}\right)+\widehat{\eta}_{\mathrm{QM}},
$$

where $\mathrm{W}(\cdot)$ is the Lambert $\mathrm{W}$ function [5]. Our third estimator applies the statistically principled

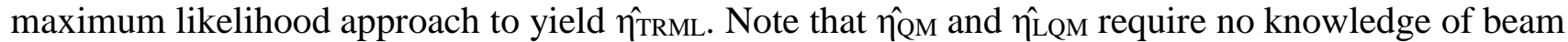
current $\lambda$. We have found $\hat{\eta}$ TRML in most cases outperforms our other estimates when $\lambda$ is known [6]. In general, when $\lambda$ is imperfectly known, $\hat{\text { LQM }}$ outperforms $\hat{\eta_{\text {TRML }}}$ in the low SE yield, high beam current conditions of SEM.

In Figure 1, we evaluate TR methods $\hat{\eta}_{\mathrm{TR}}$ in five different scenarios. Synthetic data was produced using existing micrographs as ground truth images [7]. Beam current time series were generated according to a first-order Gaussian autoregressive model with mean $\tilde{\lambda}$ and correlation coefficient $\rho$ between neighboring pixels. Higher values of $\rho$, as in rows 3 and 5, model slower beam current variation and lower values, as in rows 1 and 4, model faster variation. Note the thicker stripe artifacts in rows 3 and 5, and the narrower stripes in rows 1 and 4. In rows 1-3, we simulate HIM conditions using ground truth images scaled for $\eta \in[2,8]$ [8], and test $\hat{\eta}$ TR $=\hat{\eta T R M L}$. Rows 4-5 model SEM with lower $\eta$ values: $\eta \in$ $[0.6,2][9]$, and evaluate $\hat{\eta_{\mathrm{TR}}}=\hat{\eta_{\mathrm{LQM}}}$. We compare the time-resolved method $\hat{\eta_{\mathrm{TR}}}$ to $\hat{\eta}_{\text {baseline }}$ and the Fourier-domain filtering procedure described in [2], which we denote $\hat{\eta}_{\mathrm{FT}}$. In this method, a 2D discrete

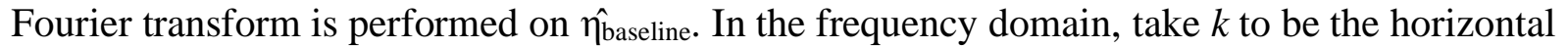
frequency index and $u$ to be the vertical frequency index; $(k, u)=(0,0)$ is the coefficient that describes the zero frequency image component. Following the procedure in [2], we null all coefficients that satisfy both $|k| \leq w$ and $|u|>h$ and then apply the inverse transform. In each example, filter parameters $w$ and $h$ were manually selected to yield optimal performance of $\hat{\eta}$ FT.

As shown in Figure 1, the TR method $\hat{\eta}_{\mathrm{TR}}$ achieves lower mean-squared error (MSE) than both $\hat{\eta}_{\text {baseline }}$ and $\hat{\eta_{\mathrm{FT}}}$ in all scenarios, regardless of the speed of current variation. Note that unlike the TR methods, $\hat{\eta}_{\text {FT }}$ requires the selection of filter parameters based on both current variation speed and image content. Gains of $\hat{\eta_{\mathrm{TR}}}$ over $\hat{\eta_{\mathrm{FT}}}$ are especially pronounced in the fast-variation scenarios, and in image regions where the underlying sample has horizontal structure (e.g., row 3). In Figure 2, we show the difference between ground truth $\eta$ and estimates $\hat{\eta}_{\text {baseline }}, \hat{\eta_{\mathrm{FT}}}$, and $\hat{\eta_{\mathrm{TR}}}$ for the same scenario shown in Figure 1, row 3. Note the prominent stripe artifacts left in the conventional reconstruction $\hat{\eta}_{\text {baseline, }}$, the remaining or newly introduced artifacts in $\hat{\eta_{\mathrm{FT}}}$, and the general lack of large structured error in $\hat{\eta_{\mathrm{TR}}}$.

These findings illustrate that, in addition to mitigating source shot noise in particle beam microscopy, TR methods prevent the artifacts typically caused by beam current variation. 

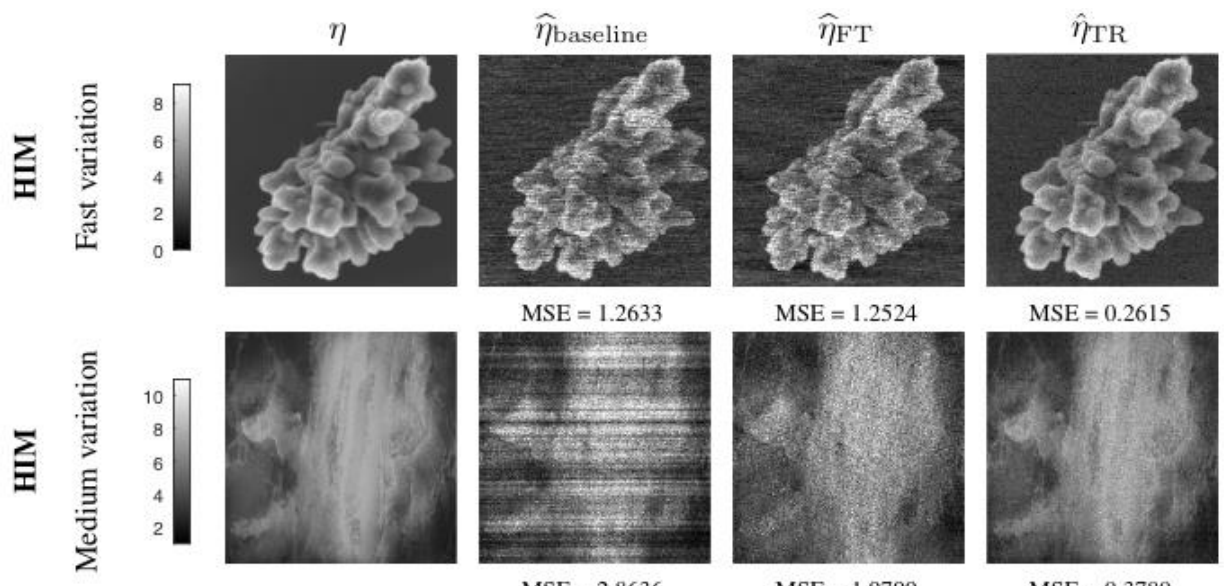

MSE $=1.2524$

MSE $=0.2615$
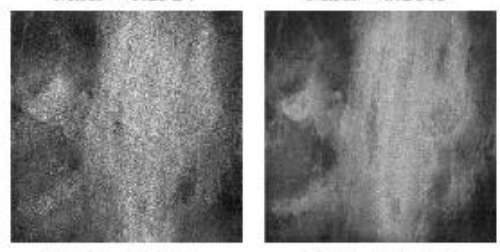

MSE $=2.8636$
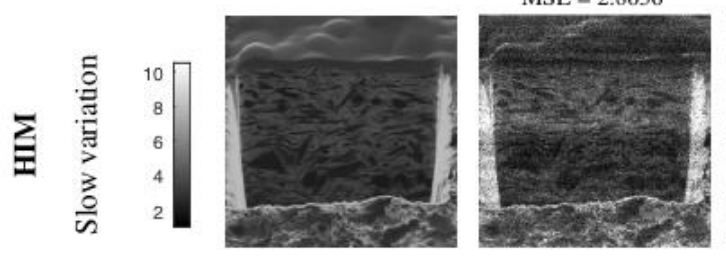

MSE $=1.9799$

MSE $=0.3780$
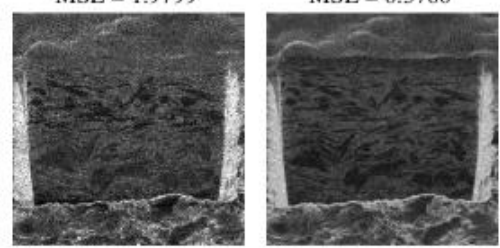

MSE $=1.6702$

MSE $=1.3681$
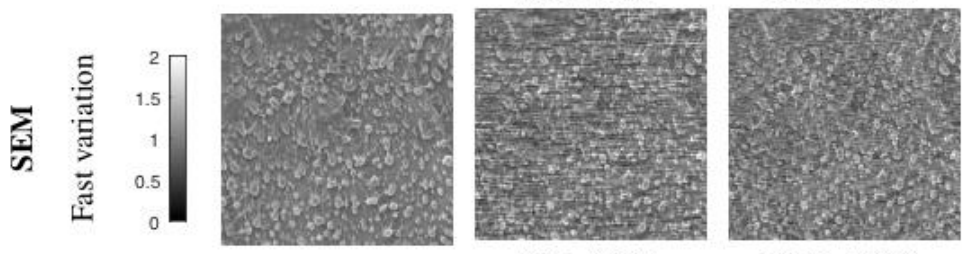

MSE $=0.0306$

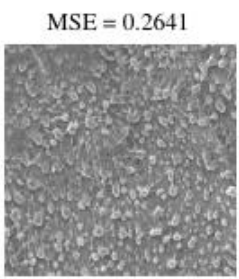

MSE $=0.0436$
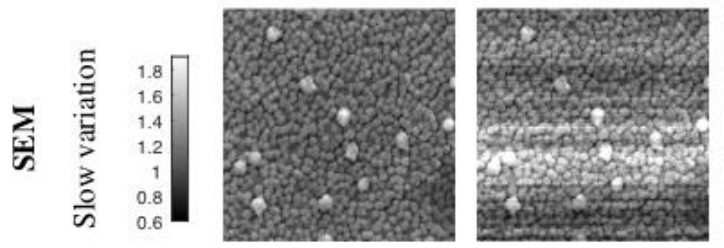

MSE $=0.0480$

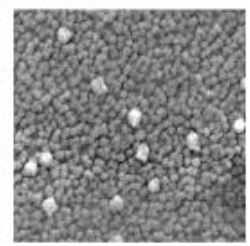

MSE $=0.0175$

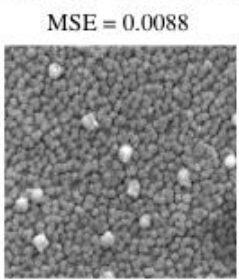

MSE $=0.0112$

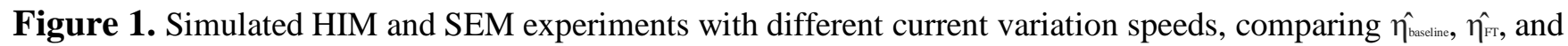

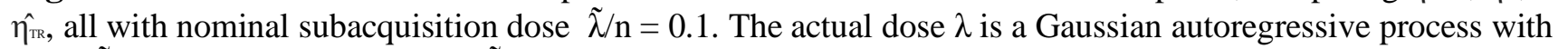
mean $\tilde{\lambda}$ and standard deviation $0.2 \tilde{\lambda}$. Correlation coefficient $\rho$ between neighboring pixels in a row is varied between test images, with lower values of $\rho$ corresponding to faster current variation. Filter parameters $(w, h)$ for $\hat{\eta}_{\mathrm{Fr}}$ were selected to give the best performance for each test image. HIM-fast: $\eta \in[2,8], \tilde{\lambda}=20$, and $\rho=0.9$, with $(\mathrm{w}, \mathrm{h})=(1,3)$. HIM-medium: $\eta \in[2,8], \tilde{\lambda}=20$, and $\rho=0.999$, with $(\mathrm{w}, \mathrm{h})=(2,2)$. HIM-slow: $\eta \in[2,8], \tilde{\lambda}$ $=20$, and $\rho=0.9999$, with $(\mathrm{w}, \mathrm{h})=(0,0)$. SEM-fast: $\eta \in[0.6,2], \tilde{\lambda}=1000$, and $\rho=0.9$, with $(\mathrm{w}, \mathrm{h})=(3$, 2). SEM-slow: $\eta \in[0.6,2], \tilde{\lambda}=1000$, and $\rho=0.9999$, with $(\mathrm{w}, \mathrm{h})=(0,0)$. 


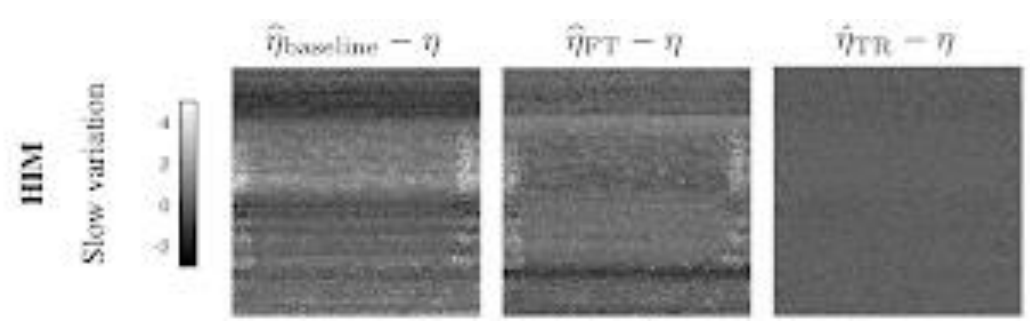

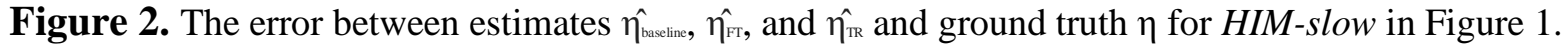

\section{References}

[1] J. Notte and J. Huang, The Helium Ion Microscope, pp. 3-30, Springer International Publishing, Cham, 2016.

[2] A. J. Barlow, J. F. Portoles, N. Sano, and P. J. Cumpson, "Removing beam current artifacts in helium ion microscopy: A comparison of image processing techniques," Microscopy and Microanalysis, vol. 22, no. 5, pp. 939-947, Oct. 2016.

[3] M. Peng, J. Murray-Bruce, K. K. Berggren, and V. K. Goyal, "Source shot noise mitigation in focused ion beam microscopy by time-resolved measurement," Ultramicroscopy, vol. 211, pp. 112948, 2020.

[4] K. S. Sim, J. T. L. Thong, and J. C. H. Phang, "Effect of shot noise and secondary emission noise in scanning electron microscope images," Scanning, vol. 26, no. 1, pp. 36-40, 2004.

[5] R. M. Corless, G. H. Gonnet, D. E. G. Hare, D. J. Jeffrey, and D. E. Knuth, "On the Lambert W function," Advances in Computational Mathematics, vol. 5, no. 1, pp. 329-359, Dec. 1996.

[6] M. Peng, J. Murray-Bruce, and V. K. Goyal, "Time-resolved focused ion beam microscopy: Modeling, estimation methods, and analyses,” arXiv:2011.08402, 2020.

[7] Thermo Fisher Scientific, five images from https://www.fei.com/uploadedimages/FEISite/Content/Image_Gallery/Images:

2016_Image_Contest/IM_20161024_Abbaspour_79_bouquet.jpg,

2014_Image_Contest/IM_20141118_GUEDJ_81_Thegoldisland.jpg, Shale_22\%20nA.jpg,

IM_20100408_Abd.\%20Jalil_4_seed_008_lg.jpg, and

2012_Image_Contest/FEI/IM_20120912_ILIESCU_88_Bubbles_lg.jpg.

[8] J. Notte, B. Ward, N. Economou, R. Hill, R. Percival, L. Farkas, and S. McVey, "An introduction to the helium ion microscope," in AIP Conference Proceedings. AIP, 2007, vol. 931, pp. 489-496.

[9] Y. Lin and D. C. Joy, "A new examination of secondary electron yield data," Surface Interface Analysis, vol. 37, no. 11, pp. 895-900, 2005. 